\title{
Femoral Fracture Caused by Removal of Femoral Intramedullary Nail Made of Stainless Steel
}

\author{
Mitsuru Aizawa $^{\text {a }}$, Tetsuya Jinno ${ }^{\text {b, e }}$, Hideki Nanke ${ }^{c}$, Kenshi Ishii ${ }^{d}$, Sadaomi Kawachic
}

\begin{abstract}
This is to report a case of femoral fracture caused by removal of a femoral intramedullary nail. The patient was a 28 -year-old male. We performed intramedullary nailing for his femoral shaft fracture with an interlocking femoral nail made of stainless steel with fluted structure and roughened surface. The nail was removed 2 years and 5 months later, and fresh fracture lines were found in the postremoval radiograph. We suspected there existed problems with the structure of the intramedullary nail as well as late removal of the nail.
\end{abstract}

Keywords: Femoral intramedullary nail; Nail removal; Refracture

\section{Introduction}

The treatment of long-bone fractures with intramedullary nailing has been a common method, and there have sporadically appeared reports about difficulties on removal of the internal fixator devices [1-7]. Although fractures during removal of intramedullary tibial nails have been reported [1-4], there have been few reports of difficult removal for femoral nails [5-7] and no reports of fracture during removal of a femoral intramedullary nail, to our knowledge. A case of femoral fracture during removal of a femoral intramedullary nail is presented. The fracture appeared to be closely tied in with the structure of the femoral intramedullary nail.

Manuscript accepted for publication January 14, 2015

aNagano Orthopaedic Clinic, 5-26-23 Yoshida, Nagano City, Nagano 3810043, Japan

bDepartment of Orthopaedic Surgery, Tokyo Medical and Dental University, 1-5-45 Yushima, Bunkyo-ku, Tokyo 113-8519, Japan

'Department of Orthopaedic Surgery, JA Toride Medical Center, 2-1-1 Hongo, Toride-shi, Ibaraki 302-0022, Japan

dDepartment of Orthopaedic Surgery, Saitama Red Cross Hospital, 8-3-33 Kamiochiai, Chuo-ku, Saitama City, Saitama 338-8553, Japan

${ }^{e}$ Corresponding Author: Tetsuya Jinno, Department of Orthopaedic Surgery, Tokyo Medical and Dental University, 1-5-45 Yushima, Bunkyo-ku, Tokyo 113-8519, Japan. Email: jinno.orth@tmd.ac.jp

doi: http://dx.doi.org/10.14740/jmc2059w

\section{Case Report}

A 28-year-old man sustained a transverse fracture on his right femur while playing soccer. A week after the injury, intramedullary interlocking nailing was performed using a Zimmer (Warsaw, IN) ZMS ${ }^{\circledR}$ femoral nail. An intramedullary nail, 10 $\mathrm{mm}$ in diameter, was inserted after $11 \mathrm{~mm}$ reaming according to the standard reaming-nailing technique. The operation was completed without any trouble, and the postoperative radiograph showed favorable reduction (Fig. 1). Postoperative course also was uneventful, and he did not visit the hospital over a period of more than 1.5 years after the bone union.

Two years and 5 months after the initial operation, the nail was removed at the patient's request. The radiograph immediately before the removal showed complete healing of the fracture with an anatomic alignment and without any hardware failure (Fig. 2). Following removal of interlocking screws, the intramedullary nail was tried to be extracted. The nail started to be pulled out with relatively little resistance at first, but considerably greater resistance was encountered after it was taken out several centimeters. After an addition of harder blows, the resistance suddenly disappeared and the nail was pulled out

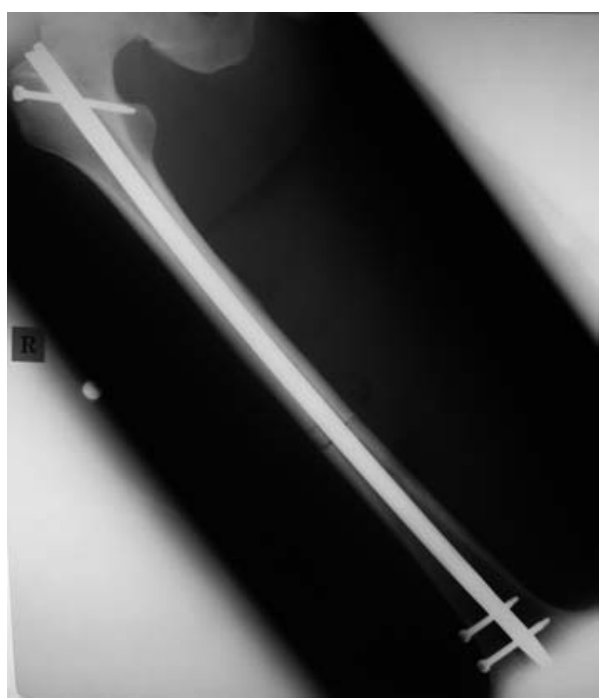

Figure 1. A postoperative AP radiograph showing anatomical reduction. 


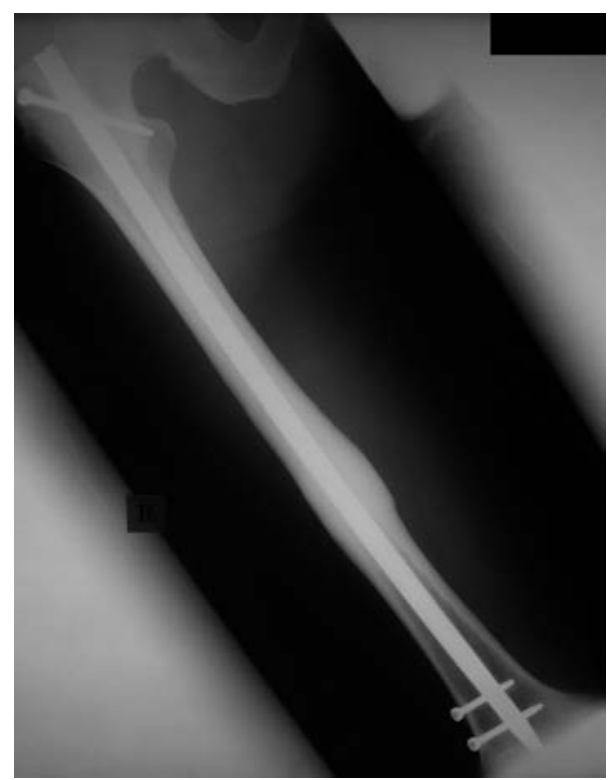

Figure 2. An AP radiograph taken immediately before the nail removal showing complete union of the fracture.

completely without stopping. There was no breakage in the removal instruments and the nail. However, the radiographs taken immediately after the removal demonstrated several new, longitudinal fracture lines proximal to the primary fracture site (Fig. 3). A CT image showed fracture lines that radiated in three directions (posterior, medial, and lateral) and a narrow, newly formed intramedullary bone that was the same in configuration as the nail surface (Fig. 4). The fracture was treated conservatively with protected weight-bearing. In the fifth postoperative month, the patient could tolerate full weight-bearing and no

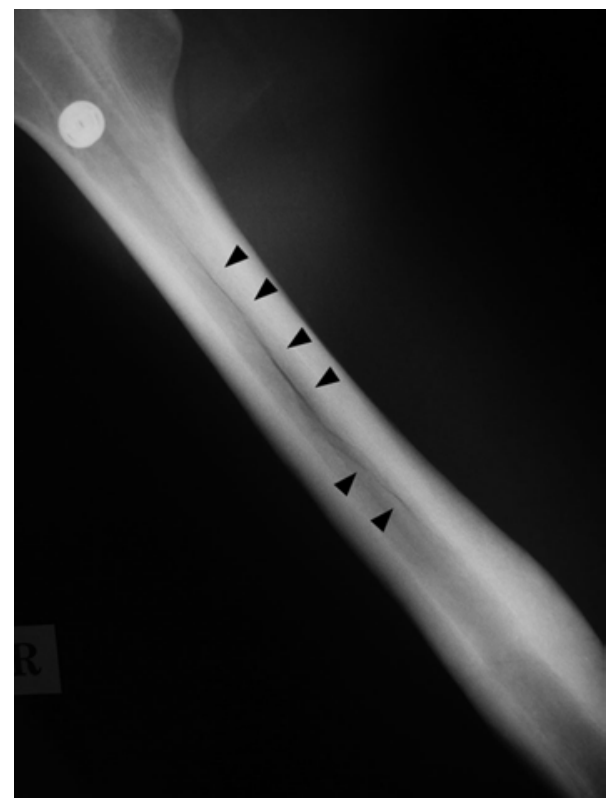

Figure 3. An AP radiograph taken immediately after the nail removal showing longitudinal fracture lines (arrows).

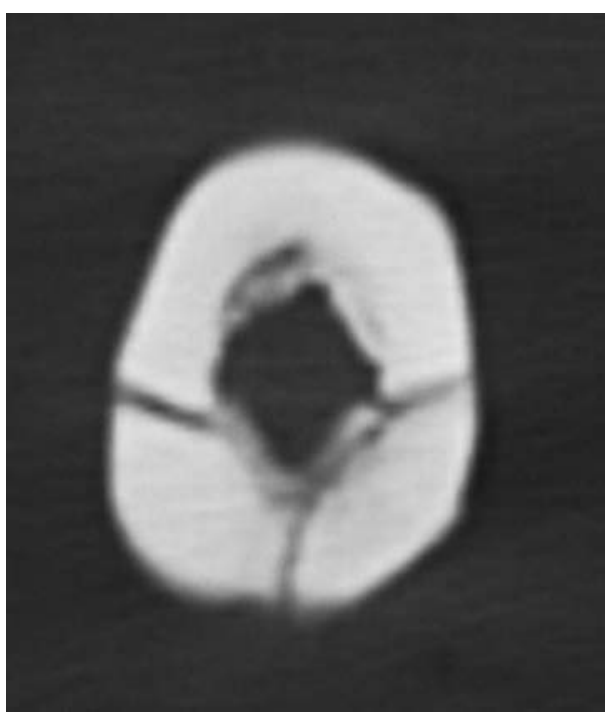

Figure 4. A CT image showing fracture lines that radiate in three directions (posterior, medial, and lateral) and a narrow, newly formed intramedullary bone that is the same in configuration as the nail surface.

significant functional disorders were observed thereafter. The patient gave his informed consent prior to being included into this case report.

\section{Discussion}

The Zimmer ZMS ${ }^{\circledR}$ femoral nail is made of stainless steel. The entire surface of the nail is treated with shot-peening to enhance the strength and corrosion resistance of the nail. It has flutes in the four directions to provide space for revascularization and to enhance fixation by four-point cortical contact. Nails which are larger in diameter (12 - $16 \mathrm{~mm}$ ) have a slot anteriorly instead of a flute to increase the flexibility of the nail. The flutes stop at the level above the distal holes for interlocking screws to preserve the nail strength, and the cross-sectional shape of the distal end of the nail, several centimeters in length, differs from the upper part of the nail, being cylindrical except the thin anterior slot (Fig. 5).

In postoperative $\mathrm{CT}$, the fracture lines were observed in three directions (posterior, medial, and lateral) of the femur. There existed a mature bone, which was newly formed in

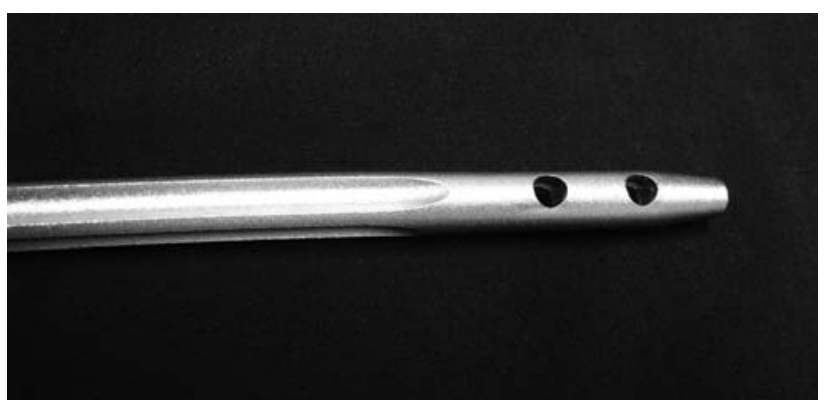

Figure 5. $\mathrm{ZMS}^{\circledR}$ femoral nail (distal end). 
the same configuration as the cross-sectional shape of the intramedullary nail. These findings suggest that the mechanism of the fracture is closely tied in with the structure of the nail stated above. That is, the mature, hard bone which was formed in the three flutes was hit against the distal cylindrical part of the nail at the distal end of the flutes when the nail was extracted, producing strong resistance, and eventually giving rise to a fracture. Seligson et al [5] reported two cases of irremovable interlocking femoral nails, including one case of $13 \mathrm{~mm}$ diameter $\mathrm{ZMS}^{\circledR}$ femoral nail, and stated that the bone overgrowth into the slot of the nail might have prevented the distal, unslotted end of the nail from being extracted from the medullary canal. They recommended the use of nails with closed and constant cross-sectional designs to prevent the problem of difficult removal. Although the $10 \mathrm{~mm}$-diameter $\mathrm{ZMS}^{\circledR}$ femoral nail used in our case did not have the slot and had a closed cross-section, it had flutes that stopped at the level above the distal holes for interlocking screws. Our case suggested the importance of the constant cross-sectional design to prevent the difficulties and the fracture on nail removal.

There have been some reports of fracture on removal of tibial intramedullary nails [1-4]. Takakuwa et al [1] reported four cases of fracture on removal of an ACE tibial nail, and stated that $5^{\circ}$ bend at the distal tip and the posterior slot which stopped at the level of the distal bend as well as late retrieval of the nail contributed to the fracture. Moreover, skepticism was brought against the necessity of reaming to use nails of larger diameter [8, 9]. Im and Lee [4] also reported three cases of fracture on removal and two cases of inability of removal of ACE tibial nails, and stated that younger age of the patient was significantly associated with the difficulty in nail removal, comparing with 30 cases of uneventful removal. Problems of the inconstant cross-sectional design, the late removal, the reaming, and the young age of the patient could be considered as contributory factors to the fracture on removal also in our case.

Although the nail used in our case was made of stainless steel, which is known as less osteoconductive material than titanium and its alloys, postoperative CT demonstrated abundant bone formation along the nail surface. This might be related to the micro-texture of the nail surface, in addition to the late removal and the patient age. The surface of the nail was not smooth because of the shot-peening applied for strength and corrosion resistance. It is known that, when the surface of a titanium implant is roughened, bone apposition onto the implant surface and bone-implant interfacial strength is increased [10]. This property seems not peculiar to titanium alloy [11], and it is possible that the roughened surface of the stainless steel nail accelerated osteoconduction on the nail surface. Unlike the surface of the metal implants for cementless arthroplasties, the surface of the metals for osteosynthesis would not have to be highly osteoconductive.

We were unable to find any previous reports on cases of the femoral fracture that occurred during removal of the intramedullary femoral nail [12-14]. Husain et al [12] reviewed their 45 cases of femoral nail removal, and did not find particular difficulties in late removal of titanium nails as well as stainless steel nails. The titanium nail in their report was a fluted nail with a satin surface, and the stainless steel nail was a slotted nail with a polished surface. Thus, in addition to the macrotexture such as cross-sectional design, the surface roughness of the nail could be a significant contributory factor to the fracture on nail removal.

The succeeded model of the intramedullary nail from the same manufacturer $\left(\mathrm{M} / \mathrm{DN}^{\circledR}\right.$ Femoral Nail, Zimmer, $\left.\mathrm{IN}\right)$ is a stainless steel nail with a polished surface, and also the flutes do not stop above the distal holes, extending almost to the distal end of the nail.

Although some previous studies have indicated that the removal of intramedullary femoral nails should be restricted in asymptomatic patients $[13,14]$, the nail removal might be useful for symptomatic patients [15]. In Asian countries, patients prefer the removal of metallic hardware also for cultural reasons $[1,4]$.

When internal fixation is to be done with an intramedullary nail and its removal is to be expected, we consider it necessary to choose a nail whose cross-sectional shape does not differ at levels and whose surface is not rough. At removal, the structure of the nail as well as the period that extends to the nail removal should be considered.

\section{Conflict of Interest}

The authors have received nothing of value, and no financial support of this study has occurred. The authors declare that they have no conflict of interest. The device that is the subject of this manuscript has been approved by Ministry of Health, Labour and Welfare, Japan.

\section{References}

1. Takakuwa M, Funakoshi M, Ishizaki K, Aono T, Hamaguchi H. Fracture on removal of the ACE tibial nail. J Bone Joint Surg Br. 1997;79(3):444-445.

2. Jones DHt, Schmeling G. Tibial fracture during removal of a tibial intramedullary nail. J Orthop Trauma. 1999;13(4):271-273.

3. Folwaczny EF, Sturmer KM. Unusual complications during removal of intramedullary nails after lower leg fractures. Tibial shaft fissure and inability to remove the nail. Eur J Trauma. 2001;27(4):184-190.

4. Im GI, Lee KB. Difficulties in removing ACE tibial intramedullary nail. Int Orthop. 2003;27(6):355-358.

5. Seligson D, Howard PA, Martin R. Difficulty in removal of certain intramedullary nails. Clin Orthop Relat Res. 1997;340:202-206.

6. Bombaci H, Gorgec M. Difficulty in removal of a femoral intramedullary nail: the geometry of the distal end of the nail. Yonsei Med J. 2003;44(6):1083-1086.

7. Star AM, Whittaker RP, Shuster HM, Duda J, Menkowitz E. Difficulties during removal of fluted femoral intramedullary rods. J Bone Joint Surg Am. 1989;71(3):341-344.

8. Sohngen G. Fracture on removal of the ACE tibial nail. J Bone Joint Surg Br. 1998;80(1):182.

9. Lob G, Andress HJ, Gradl G. Fracture on removal of the ACE tibial nail. J Bone Joint Surg Br. 1998;80(3):557. 
10. Feighan JE, Goldberg VM, Davy D, Parr JA, Stevenson $\mathrm{S}$. The influence of surface-blasting on the incorporation of titanium-alloy implants in a rabbit intramedullary model. J Bone Joint Surg Am. 1995;77(9):1380-1395.

11. Jinno T, Goldberg VM, Davy D, Stevenson S. Osseointegration of surface-blasted implants made of titanium alloy and cobalt-chromium alloy in a rabbit intramedullary model. J Biomed Mater Res. 1998;42(1):20-29.

12. Husain A, Pollak AN, Moehring HD, Olson SA, Chapman MW. Removal of intramedullary nails from the femur: a review of 45 cases. J Orthop Trauma. 1996;10(8):560-
562.

13. Miller R, Renwick SE, DeCoster TA, Shonnard P, Jabczenski F. Removal of intramedullary rods after femoral shaft fracture. J Orthop Trauma. 1992;6(4):460-463.

14. Gosling T, Hufner T, Hankemeier S, Zelle BA, MullerHeine A, Krettek C. Femoral nail removal should be restricted in asymptomatic patients. Clin Orthop Relat Res. 2004;423:222-226.

15. Toms AD, Morgan-Jones RL, Spencer-Jones R. Intramedullary femoral nailing: removing the nail improves subjective outcome. Injury. 2002;33(3):247-249. 